\title{
Une anthologie des musiques sibériennes
}

\author{
Mireille Helffer
}

\section{(2) OpenEdition \\ Journals}

Édition électronique

URL : http://journals.openedition.org/ethnomusicologie/934

ISSN : 2235-7688

Éditeur

ADEM - Ateliers d'ethnomusicologie

Édition imprimée

Date de publication : 1 décembre 1997

Pagination : 339-341

ISBN : 2-8257-0579-9

ISSN : $1662-372 X$

Référence électronique

Mireille Helffer, "Une anthologie des musiques sibériennes », Cahiers d'ethnomusicologie [En ligne],

10 | 1997, mis en ligne le 06 janvier 2012, consulté le 01 mai 2019. URL : http://

journals.openedition.org/ethnomusicologie/934

Ce document a été généré automatiquement le 1 mai 2019.

Tous droits réservés 


\title{
Une anthologie des musiques sibériennes
}

\author{
Mireille Helffer
}

\section{RÉFÉRENCE}

Sibérie 1 : Nganasan. Chants chamaniques et narratifs de l'Arctique sibérien. BUDA Records, coll. Musique du Monde 92564-2 / ADE 670

Sibérie 2 : Sakha.Yakoutie : Epopées et Improvisations. BUDA Records, coll. Musique du Monde 92565-2 / ADE 670

Sibérie 3: Čukč, Even, Jukaghir. Kolyma: Chants de nature et d'animaux. BUDA Records, coll. Musique du Monde 92566-2 / ADE 670

Sibérie 4 : Korjak. Kamtchatka : tambours de danse de l'Extrême-Orient sibérien. BUDA Records, coll. Musique du Monde 92598-2 / ADE 670

Sibérie 5 : Nanaj, Oroc, Udege, Ulc. Chants chamaniques et quotidiens du bassin de l'Amour. BUDA Records, coll. Musique du Monde 92671-2 / AD 761

1 Réaliser une anthologie de la musique des peuples autochtones de Sibérie en quinze CD, tel est l'objectif que s'est fixé Henri Lecomte, dont on connaît la passion pour les musiques dites traditionnelles. Appuyée par des personnalités scientifiques françaises et russes, cette entreprise, qui peut paraître démesurée, a commencé en 1992, et a été soutenue dès ses débuts par l'UNESCO ; ultérieurement, la Direction de la Musique et de la Danse du ministère français de la Culture et de la Francophonie, comprenant l'originalité d'un tel projet, a accordé à l'auteur une allocation lui permettant de poursuivre sa recherche. Depuis lors, en dépit des difficultés administratives et des rigoureuses conditions climatiques qu'on peut imaginer, les missions de terrain se sont succédées, en hiver comme en été. $\mathrm{H}$. Lecomte s'étend peu sur les conditions dans lesquelles il a pu réaliser ces enregistrements mais, à la lecture des notices, on comprend que les minorités autochtones de Sibérie ( $5 \%$ de la population), réparties sur plus de 12 millions de $\mathrm{km}^{2}$ et 
nomades pour la plupart, ont été sédentarisées de force par le pouvoir soviétique et ont fait l'objet de déportations et de "regroupements » dans des villages où elles étaient supposées recevoir plus aisément les bienfaits de la civilisation. Dans ces régions reculées du grand Nord, il y a eu certes création d'écoles, mais, corrélativement, abandon progressif des langues vernaculaires dont le nombre de locuteurs va diminuant de jour en jour. Ces conditions rendent d'autant plus précieux les nombreux enregistrements réalisés auprès d'informateurs le plus souvent âgés (certains d'entre eux sont nés à la fin du XIXe s.) et ayant connu les conditions de vie anciennes : élevage des rennes, chasse, pêche.

2 A l'heure qu'il est (printemps 1997), grâce à l'audacieuse politique éditoriale de Buda Records, cinq premiers $\mathrm{CD}$, consacrés à dix ethnies parmi les quelques trente réparties dans l'immensité sibérienne, sont disponibles. Si l'on s'en tient aux données d'un recensement de 1989, les unes, comme les Sakha (anciennement Yakoutes) comptent près de 400000 individus, tandis que les autres sont beaucoup moins nombreuses : 17200 Even, 15200 Čukč, 12000 Nanaj, 8000 Korjak, 3200 Ulč, 1469 Udēgē, 1100 Jukaghir, 900 Oroč.

3 Le matériel sonore collecté est abondant : 140 pièces recueillies aussi bien en Yakoutie/ République Sakha (Sibérie 2) qu'au centre du grand Nord sibérien (Sibérie 1), dans la vallée de l'Amour (Sibérie 5) ou de la Kolyma (Sibérie 3), dans la péninsule du Kamtchatka (Sibérie 4$)^{1}$; il illustre principalement les genres de musique vocale pratiqués par les uns et les autres, et exécutés pour la plupart en solo; ajoutons que, sur chaque $C D$, les enregistrements sont classés par groupe ethnique et par informateur.

On sera sensible à la richesse des expressions féminines, majoritaires dans les cinq $\mathrm{CD}$ : berceuses (Sibérie $4: 12$ et 24 et Sibérie $5: 1,16,19,26$ ), chants personnels (Sibérie 4), chants de cueillette (Sibérie $5: 4,15,29$ ), chants chamaniques, accompagnés au tambour sur cadre à une peau (Sibérie $1: 12$; Sibérie 4 ; Sibérie $5: 8,27,28,31$ ), éloges tojuk (Sibérie $2: 18-19)$, extraits d'épopée.

5 Tout à fait saisissante apparait la variété des techniques vocales: si aucune des populations citées ne pratique le chant diphonique illustré par leurs voisins touvas ou mongols, elles n'en connaissent pas moins des raffinements et des jeux vocaux parmi lesquels on signalera diverses formes du parlé/chanté, les raclements de gorge (khabarba yryata des Sakha, Sibérie 2: ou tumun khontol des Jukhagir, Sibérie 3:39) et les claquements de langue (tanalaï yryata) des Sakha (Sibérie 2), le "parlé précipité» (cabyrgakh) (Sibérie $2: 16$ ), les imitations du chant des oiseaux ou du cri du renne (Sibérie $3: 11$ et $12,27,28) \ldots$

6 Quant aux instruments de musique, à l'exception du tambour chamanique, leur usage demeure limité, qu'il s'agisse de la guimbarde en métal (khomus des Sakha, kunkaj des Udēgē) souvent utilisée avec une grande virtuosité, de différents types de vièles (Sibérie $2: 3$ à 10 ; Sibérie $5: 21$ ), des trompes en écorce des Udēgē (Sibérie $5: 18$ ).

7 La présentation est soignée mais sommaire : les notices bilingues français-anglais ( $35 \mathrm{pp}$.) fournissent les informations indispensables relatives au lieu (une carte schématique accompagne chaque $\mathrm{CD}$ ) et à la date des enregistrements, au nom des informateurs (avec leur date de naissance), au titre de la pièce en traduction, aux genres représentés, mais ne comportent aucune donnée d'ordre musical. Les photos couleur des principaux informateurs, placées en couverture ou groupées au centre de la plaquette, sont certes utiles ; mais, en raison sans doute du caractère provoqué des enregistrements (aucun ne semble avoir été fait véritablement « en situation »), elles sont souvent empreintes d'une 
raideur conventionnelle; certaines d'entre elles prouvent néanmoins que le fameux tambour chamanique est toujours présent dans ces sociétés, aussi bien chez les Nganasan (Sibérie 1: photo de couverture) que chez les Čukč (Sibérie $3:$ photo de couverture), les Korjak du Kamtchatka (Sibérie $4:$ photo de couverture et photos 1 et 4), les Nanaj (Sibérie 5 : couverture) ou les Ulč (Sibérie $5:$ photos 1 et 4 ).

8 L'étude musicologique de ces musiques austères reste donc à entreprendre, mais la simple écoute des documents collectés ouvre des perspectives comparatives fascinantes avec les musiques mieux connues des Ainous (désormais regroupés dans l'île japonaise de Hokkaido), des Inuits d'Alaska, du Canada et du Groenland, bref avec ces musiques « circumpolaires » selon le terme employé par Jean-Jacques Nattiez. Pour un tel travail, il faudra disposer des textes vernaculaires des chants qui ont été recueillis simultanément et dont on peut espérer qu'ils seront inclus dans l'ouvrage collectif annoncé auquel devraient collaborer de nombreux spécialistes russes, linguistes et musicologues.

9 Dans l'attente du prochain CD qui sera consacré aux Nikhs de l'île de Sakhaline, il faut savoir gré à Henri Lecomte d'avoir mis sur pied un tel projet et d'en assurer la continuation; grâce à lui et à son patient travail de collectage, la communauté scientifique et tous ceux qui s'intéressent aux musiques du Grand Nord disposent d'un matériel exceptionnel. Il faut aussi saluer l'effort consenti par Buda Records pour promouvoir une musique dont la sobriété n'est sans doute pas près de séduire les foules !

\section{BIBLIOGRAPHIE}

DIRMID, R.F. Collis (ed.), 1990, Arctic Languages. An Awakening. Paris : UNESCO

TAE-GON Kim, HOPPAL Mihaly (eds.), 1995, Shamanism in Performing Arts. Budapest : Akademiai Kiado.

CHARRIN Anne-Victoire (sous la direction de), 1994, Les Sibériens. De Russie et d'Asie, une vie, deux mondes. Paris : Editions Autrement Série Monde HS nº 78.

\section{NOTES}

1. Pour situer régions et populations, on se reportera utilement aux cartes publiées dans Les Sibériens Editions Autrement, H.S. № 78, publié en oct.1994 : pp. 16-19. 